\title{
Entwicklung eines akustischen Füllstandsensors für mobile Schüttgutsilos
}

\author{
Dipl.-Ing. Paaranan Sivasothy, B. Sc. Hüseyin Cavuldak, M. Sc. Vitali Rjasanzew, Prof. Dr.-Ing. Jörg \\ Seewig \\ Lehrstuhl für Messtechnik und Sensorik, Technische Universität Kaiserslautern, \\ 67663 Kaiserslautern, Gottlieb-Daimler-Str. 44, Deutschland
}

\section{Zusammenfassung}

Starre Silobehälter stehen oft unter Druck und sind mit Gefahrstoffen gefüllt. Der Füllstand kann in der Regel nicht per Sichtprüfung bestimmt werden. Daher wird beispielsweise der Füllstand von mobilen Schüttgutsilos, die häufig auf Baustellen eingesetzt werden, von einer Arbeitskraft überprüft, der einen Stein gegen das Silo wirft und den nicht sichtbaren Silohohlraum anhand des Klangs abschätzt. Allerdings ist diese Methode subjektiv und weist erfahrungsgemäß große Fehler auf. Das vorgeschlagene Verfahren "Silopic" soll das Prinzip der "Steinwurfmethode" maschinell umsetzen. Eine Sensoreinheit mit einem Schlagwerk gibt einen mechanischen Impuls auf die Siloaußenwand und der entstehende Klang wird mit einem Mikrofon aufgezeichnet. Aus der akustischen Impulsantwort soll ein Kennwert berechnet werden, der zur Abschätzung des Füllstands geeignet ist. Weiterhin wird in dieser Arbeit der Einfluss der eingebrachten Erregerenergie, der Sensorpositionierung auf der Mantelfläche des Silos und Mikrofonabstandes auf das Messverfahren untersucht.

Keywords: Füllstandmesstechnik, Schüttgut, Silo, Wavelet-Transformation, Akustik,

\section{Motivation}

Seit vielen Jahrzehnten sind verschiedenste Füllstandsensoren für Gase und Flüssigkeiten etabliert. Fluide können dabei als eine homogene Masse betrachtet werden. Füllstandmesssysteme für Schüttgüter sind jedoch seltener, komplexer und entsprechend teurer. Der Grund hierfür liegt in der komplexen Natur von Schüttgütern, die sich aus einer immensen Menge an heterogenen Festkörperteilchen zusammensetzen.

Gerade beim Einsatz von mobilen Schüttgutsilos, wie sie auf den meisten größeren Baustellen vorzufinden sind, wird daher auf jegliche Art von Füllstandsensorik verzichtet. Stattdessen wird der Füllstand manuell abgeschätzt. Hierzu kommen in der Regel zwei unterschiedliche Verfahren zum Einsatz:

\section{Methode 1: Das Abklopfen mit einem} Besenstiel

Eine gängige Form der Füllstandmessung bei Schüttgutsilos ist das Abklopfen mit einem Besenstiel. Eine Arbeitskraft der Baustelle führt hier mehrere Schläge auf die Siloaußenwand aus, wobei er dabei mit dem Schlag örtlich vertikal nach oben wandert. Wandelt sich die dabei resultierende akustische Impulsantwort subjektiv von einem tiefen, dumpfen Klang zu einem hellen, lauten Klang, so gilt die Grenzschicht zwischen Schüttgutmaterial und dem Hohlraum im inneren des Silos als gefunden. Auf Basis dieser Füllhöhe versucht die Arbeitskraft nun den Füllstand in Tonnen abzuschätzen und diese Information zum Zwecke der Nachbestellung an den Bauherrn weiterzugeben.

Methode 2: Das Steinwurf-Verfahren

Eine schnellere Methode, die gerade von erfahreneren Mitarbeitern bevorzugt wird, ist die Steinwurfmethode. Die Arbeitskraft wirft einen Stein auf einen bestimmten Bereich der Siloaußenwand. Auf Grundlage seines erfahrungsbasierten Wissens über den Zusammenhang zwischen Füllstand und akustischer Impulsantwort versucht die Arbeitskraft den tatsächlichen Füllstand abzuschätzen.

Bei der Verwendung dieser beiden manuellen Verfahren zur Füllstandabschätzung stellen sich gewisse Verluste ein. Bei der ersten vorgestellten Methode kann relativ gut die Füllstandhöhe bestimmt werden, sofern der Füllstand im für den Arbeiter zugänglichen 
Bereich liegt. Äußerst kritisch ist jedoch die subjektive Abschätzung des Füllgewichts auf Basis der Füllstandhöhe. Vor allem aufgrund des Trichtergeometrie und der Einbauten im Siloinneren ist diese Zuordnung nicht vollständig linear und daher ohne eine detaillierte Berechnung kaum zuverlässig zu erbringen.

Die zweite vorgestellte Methode basiert vollkommen auf der Erfahrung der ausführenden Arbeitskraft. Die Qualität der Messung hängt somit stark von zeitlich veränderlichen Faktoren wie Alter, Gesundheit und Tagesform der einzelnen Arbeitskraft ab. Daher ist diese Methode als hochgradig unzuverlässig zu beurteilen, ungeachtet der zeitlichen Einsparung, die diese Methode grundsätzlich mit sich bringen kann. Zudem entstehen durch die Steinwurfmethode erhebliche Schäden auf der Mantelfläche des Silos, was regelmäßig kostenintensive Wartungsarbeiten mit sich zieht.

Bei manuellen Messungen wird die zuverlässige und regelmäßige Durchführung durch eine Arbeitskraft vorausgesetzt. Im alltäglichen Stress der wechselhaften Baustellenarbeiten ist dies jedoch relativ unrealistisch.

Durch das Leerlaufen eines Silos kann es zum Stillstand von Bauarbeiten kommen. Dieser Stillstand zieht eine Stagnation des Projektfortschritts mit sich, währenddessen die zeitvariablen Kosten wie Mitarbeiterlohn und Miete für Arbeitsmaschinen weiterhin entrichtet werden müssen. Der deutsche Bundesgerichtshof fällte im Oktober 2018 ein Urteil, wonach stillstandbedingte Mehrkosten durch den Auftragnehmer, also dem Bauunternehmen, übernommen werden müssen. Durch den Wunsch diesem Risiko vorzubeugen, steigt innerhalb der Baubranche die Nachfrage nach einer automatisierten kontinuierlichen Füllstandüberwachung erheblich an.

\section{Stand der Füllstandmesstechnik}

Die Einsatzbedingungen für die Schüttgutsilos gelten als recht rau [1]. Innerhalb des metallenen Behälters kommt es bei Befüll- und Entleervorgängen zu einer enormen Staubbildung. Am Sensor anhaftendes Material ist eine häufige Ursache für fehlerhafte Messungen. Darüber hinaus liegt aufgrund der Anzahl an laufenden Maschinen und Arbeiten eine enorme akustische Belastung vor. Weiterhin führt die mechanische Beanspruchung durch bewegende Feststoffpartikel und die damit einhergehenden abrasiven Prozesse zu einer kurzen Lebensdauer von Sensoren im Innenraum des Silos [1]. Die Montage heutiger Sensoren erfordert aufwändige Nachrüstarbeiten (Aufschneiden, Einsetzen, Schweißen, Schleifen, etc.), die mitsamt den Sensorkosten i.d.R. zwischen $5.000-10.000 €$ liegen. Wird der Sensor jedoch von außen an das Silo angebracht, so ist dieser unterschiedlichsten Umgebungseinflüssen ausgesetzt.

Aufgrund der rauen Einsatzbedingungen resultieren folgende Forderungen an ein Füllstandmesssystem für mobile Schüttgutsilos: der Sensor muss

- den Füllstand stufenlos ermitteln,

- robust gegen äußere Einflüsse sein,

- nicht invasiv an das Silo montierbar sein,

- energieeffizient genug für einen Batteriebetrieb über mehrere Jahre sein,

- wartungsfrei sein und

- $\quad$ kostengünstig sein $(<1000 €)$.

Heute gibt es eine Vielzahl von Messsystemen mit unterschiedlichen Messprinzipien, wobei keins die oben beschriebenen Anforderungen vollständig und zufriedenstellend erfüllt.

Kostengünstig und hinreichend zuverlässig sind vor allem die als Grenzwertschalter verwendeten Sensoren. Als Beispiel sei hier der Drehflügelschalter genannt [2]. Dabei wird eine kleine, rotierende Welle, die in der Mantelfläche gelagert wird, von außen permanent durch einen Elektromotor angetrieben. Am anderen Ende, welches in den Innenraum des Silos ragt, befindet sich ein Drehflügel. Kann die Welle frei rotieren, so ist der Füllstand des Silos niedriger als die Montageposition des Sensors. Wird die Rotation jedoch verhindert, so ist der Füllstand höher. Als großer Nachteil des Drehflügelschalters gelten seine große Abmessung, die Montage und der Verschleiß der bewegten Teile [2].

Ein anderes Beispiel für einen Grenzschalter ist der Vibrationsschalter. Hierbei ragt eine Stimmgabel, die von außen piezoelektrisch angeregt wird, ins Innere des Silos [3] [4]. Die Stimmgabel schwingt frei mit ihrer Eigenfrequenz. Taucht die Stimmgabel jedoch in das Schüttgut ein, so kann eine signifikante Dämpfung der Schwingungsamplitude gemessen werden [3]. Der Vibrationsschalter zeichnet sich vor allem durch seine Robustheit gegenüber Ablagerungen und Anhaftungen bei staubartigem und granuliertem Schüttgut aus [3].

So robust wie sie auch sein mögen, haben die Sensordaten von Grenzwertschalter eine geringe Aussagekraft. Für die zuverlässige 
Planung der Bauarbeiten ist eine stufenlose Messung des Füllstandes erforderlich.

Die fünf gängigsten Messverfahren bei der stufenlosen Füllstandüberwachung in Schüttgutsilos sind

- Elektromagnetisches Lotsystem

- Kapazitive Sensorik

- Radar (freistrahlend, geführt)

- Radiometrie

- Ultraschall

Elektromagnetische Lotsysteme verfügen über ein Tastgewicht, welches über ein gewickeltes Messband oder -seil auf die Füllgutoberfläche herabgelassen wird [5]. Die abgewickelte Messbandlänge wird über ein Zählrad und einen dazugehörigen Induktivgeber überwacht [5]. Das Auftreffen des Tastgewichtes auf der Materialoberfläche wird durch das plötzlich abfallende Lastmoment sensorisch detektiert und anschließend der Füllstand berechnet. Elektromagnetische Lotsysteme sind vor allem für besonders große Silos mit einer Höhe von bis zu $70 \mathrm{~m}$ mit Schüttgütern wie Kalk, Steine oder Koks geeignet [5]. Die Vorzüge dieses einfachen Systems kommen vor allem bei der Entstehung von brennbaren Stäuben zum Ausdruck. Für mobile Silos ist das System u.a. wegen der Sensitivität gegenüber Drücken über 2 bar ungeeignet.

Kapazitive Messverfahren sehen ein Kondensatorgebilde aus Silowand und einer Messsonde vor [2] [5] und können bei einer Messfrequenz zwischen $20 \mathrm{kHz}$ und $2 \mathrm{MHz}$ sowohl zur Grenzwertmessung [4] als auch zur kontinuierlichen Messung [2] genutzt werden. Die Kondensatorkapazität, die direkt von der Dielektrizitätskonstante abhängig ist, variiert mit der Veränderung der Materialeigenschaften der Stoffe zwischen Silowand und der kapazitiven Messsonde [2] [4] [5]:

- Isolation der Sonde

- Silogeometrie

- Füllguttemperatur

- Körnung des Schüttgutes

- Abrasivität

- Chemische Agressivität

- Druck im Siloinneren

- Kondensatbildung

Die Kapazität wird entweder durch Resonanzschaltungen oder selbstabgleichende Wechselstrombrücken gemessen. Grundsätzlich gilt, dass mit steigendem Füllstand die Kapazität ebenfalls steigt [5].

Kapazitive Füllstandmessverfahren sind kostengünstig [6], hinreichend robust gegen Druckunterschiede [2] und ziehen gegenüber allen anderen gängigen Messsystemen den geringsten Aufwand mit sich [4]. Die Nachteile sind jedoch die Vielzahl an Einflussparametern auf die Kondensatorkapazität und der hohe Energieverbrauch.

Seit 2001 wird Radar zur Füllstandermittlung in Schüttgutsilos eingesetzt [7]. Dabei strahlt die trichterförmige Antenne (vgl. Abb. 1), die in einer kleinen Behälterstutze montiert ist [1], einen Funkwellenimpuls aus, welcher sich im Frequenzbereich zwischen $10-79 \mathrm{GHz}$ mit Lichtgeschwindigkeit ausbreitet [5] [6] [8]. Dieses Signal wird auf der Materialoberfläche, wegen des Dielektrizitätszahlunterschieds zwischen Luft und Schüttgut, reflektiert [6]. Zur Auswertung der reflektierten Funkwelle können zwei Verfahren zum Einsatz kommen:

a. Puls-Radar-Verfahren

b. Frequenzmodulierte

Dauerstrichmethode (z. engl.

Frequency Modulated Continuous Wave radar)

Beim Puls-Radar-Verfahren wird die Laufzeit vom Entsenden des Signals bis zum Empfangen des reflektierten Echosignals gemessen. Trotz des geringen Energiebedarfs und den unwesentlichen Betriebskosten [9], birgt dieses Verfahren gewisse Nachteile. Diese resultieren aus den bei Füllstandmessungen üblich kleinen Laufzeiten im Nano-Sekundenbereich $\left(10^{-9} \mathrm{~s}\right)$ [5] und der Überlappung des Sende- und Empfangssignals. Die Auflösung und Filterung der hochwertigen Auswerteelektronik muss diesen Phänomenen genügen.

Das häufiger verwendete FMCW-Verfahren sieht eine geringfügige Variation der kontinuierlichen Trägerfrequenz vor [5]. Somit kann anhand der exakten Frequenz des reflektierten Signals die Laufzeit und somit der Füllstand kontinuierlich überwacht werden.

Der Einsatz von Radar zur Füllstandüberwachung bringt eine Robustheit gegenüber Staubbildung [1] [7], Anhaftung an der Antenne und Temperatur- und Druckschwankungen mit sich [1] [5] [7]. Darüber hinaus sind der große Messbereich von bis zu $100 \mathrm{~m}$ und die Einsetzbarkeit unter dynamischen Prozessbedingungen vorteilhaft. Rein qualitativ wird die Radarfüllstandmesstechnik für Schüttgutsilos oft als das „beste" Füllstandmessverfahren gewertet [6]. Die Nachteile sind vor allem der hohe Energieverbrauch, die Anschaffungskosten und der enorme Aufwand bei der Erstinbetriebnahme [7]. Weitere Einschränkungen liegen vor, wenn die empfangenen Reflektionen des Sendesignals weniger direkt von der Schüttgutoberfläche, sondern vielmehr von Reflektionen auf Einbauten, Rührwerk, Schweißnähten, etc. herrühren (vgl. Abb. 1) [7] [8]. 


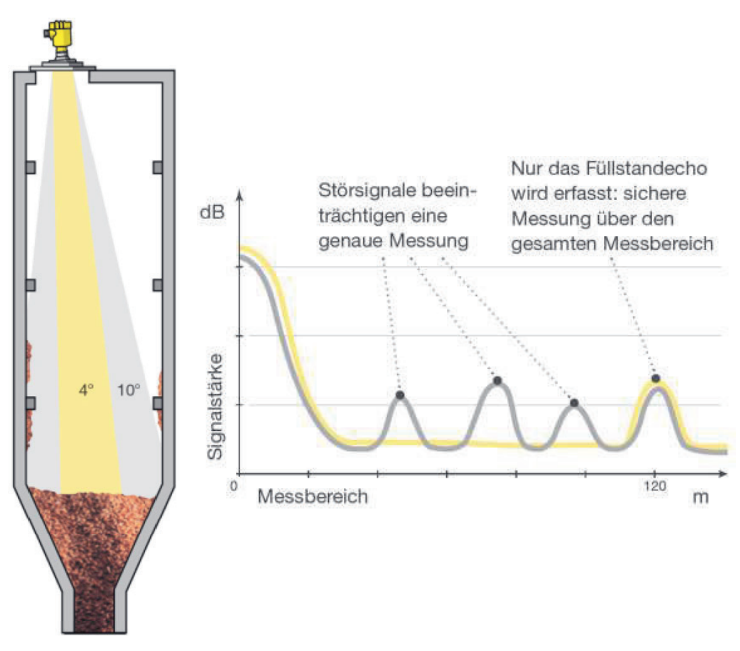

\section{Abb. 1: Radar-Füllstandmesstechnik [8]}

In solchen Fällen kann der Einsatz eines geführten Radars anstelle eines freistrahlenden Radars zielführend sein. Dabei wird die Funkwelle nicht in den freien Raum abgestrahlt, sondern in einen Metallstab, welcher im Material eingetaucht ist. Der Teil der Radarwellen, der an der Schüttgutoberfläche reflektiert wird, ermöglicht eine Laufzeitmessung [10].

In gewissen Anwendungen wird der Füllstand eines Schüttgutsilos mittels der Radiometrie ermittelt. Das Prinzip beruht dabei auf der Schwächung von Gammastrahlen durch die Masse des durchstrahlten Materials [5]. Dabei befindet sich an einer Stelle am äußeren Mantel des Silos ein Gammastrahler mit radioaktiven Isotopen und auf der anderen Seite ein Strahlenempfänger. Das GammaStrahlenbündel durchdringt das Silo bspw. diagonal durch die Mittelachse. Das Messverfahren auf Basis der Radiometrie weist in der Regel keine Einschränkungen durch Betriebsbedingungen oder Materialeigenschaften auf [5]. Jedoch ist der Einsatz von radioaktivem Material mit hohen Sicherheitsrisiken verbunden [5]. Beschaffung, Lagerung, Handhabung und Transport unterliegen strengen Auflagen. Daher kommt die kostenintensive radiometrische Messung lediglich dann zum Einsatz, wenn alle anderen Messverfahren versagen [5].

Das gängigste Messverfahren für die Füllstandüberwachung bei mobilen Schüttgutsilos ist Ultraschall. Im Gegensatz zum Radar werden hier steilflankige Schallwellenimpulse mit einer Dauer von $1 \mathrm{~ms}$ im Frequenzbereich zwischen $20 \mathrm{kHz}$ und 2 $\mathrm{MHz}$ in der Regel mittels eines piezoelektrischen, früher noch oft magnetorestriktiven Wandlers erzeugt [2] [3] [5]. Die Schallwelle kann zusätzlich durch eine akustische Linse und einer gekrümmten
Wandleroberfläche fokussiert werden [3]. Dieser Impuls breitet sich mit Schallgeschwindigkeit aus und wird an der Schüttgutoberfläche aufgrund des Dichteunterschiedes zwischen Luft und Schüttgut reflektiert [6]. Mit zunehmender Frequenz wird der Ausbreitungscharakter der Schallwelle gradlinig und sie verhält sich wie ein Lichtstrahl [2]. Daher gilt in dem Fall das Reflexions- und Brechungsgesetz aus der Optik. Wichtig ist erneut einen anwendungsspezifischen, zeitlichen Mindestabstand zwischen zwei Sendeimpulsen einzuhalten, um eine Überlagerung des Sendeimpulses mit dem Echosignal zu verhindern. Das Verfahren ist wirtschaftlich und birgt flexible Einbaumöglichkeiten [6]. Die Genauigkeit, die Wartungsfreiheit und die Robustheit gegenüber zeitabhängigen Einflussparametern begründen den dominanten Einsatz von Ultraschall [3][6]. Ultraschall versagt jedoch bei Staubbildung [7]. Es kommt zu Reflektionen an den Staubpartikeln, Einbauten und Schweißnähten und ist daher für dynamische Einsatzbedingungen kritisch. Die Temperaturabhängigkeit der Schallgeschwindigkeit bringt gerade bei Anwendungen im Außenbereich einen Laufzeitfehler mit sich [3] [6].

\section{Konzept „Silopic“}

Der in [11] vorgestellte "Silopic"-Ansatz zur Füllstandabschätzung in Schüttgutsilos beinhaltet unter anderem ein neues Sensorkonzept des Instituts für Messtechnik und Sensorik (MTS) der Technische Universität Kaiserslautern in Kooperation mit WIKON Kommunikationstechnik GmbH (WIKON) und HUEBINET Informationsmanagement $\mathrm{GmbH}$ \& Co. KG (HUEBINET) auf Grundlage der "Steinwurfmethode". Das System besteht aus einem Aktuator und einem Sensor, der von außen mit Magneten am Silomantel befestigt ist (vgl. Abb. 2). Der Aktuator ist ein elektromagnetisches Schlagwerk, das die Silowand durch einen mechanischen Impuls anregt. Dadurch wird das Silo zur Schwingung angeregt und die akustische Impulsantwort über den Sensor aufgezeichnet. Die Schwingungsdaten werden über das Mobilfunknetz an eine Cloud weitergeleitet. Dort befindet sich ein Software-Modul, das die Sounddaten auswertet. Ziel ist die Zuordnung vom Klang zu einem Füllstand. Hierzu werden die Erkenntnisse verwendet, die aus analytischen Berechnungen, numerischen Simulationen mit Kopplung der Finite- 
Elemente-Methode und der DiskreteElemente-Methode, experimentellen Tests und Ansätzen des maschinellen Lernens resultieren. Die Füllstandinformation wird dem Benutzer über eine App zur Verfügung gestellt. Diese App beinhaltet ebenfalls eine automatisierte und optimierte Logistikplanung für den Schüttgutzulieferer.

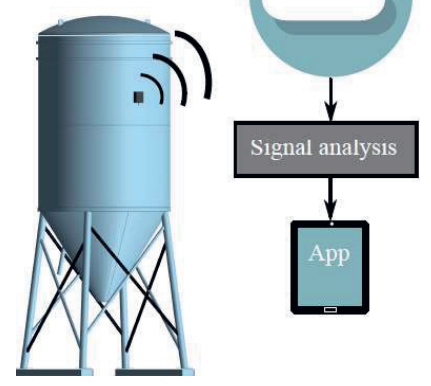

\section{Abb. 2: Sensorkonzept „Silopic“ [11]}

Im Gegensatz zu herkömmlichen Messsystemen hat das Silopic-System den Vorteil, dass die Messeinheit nicht invasiv montiert werden kann, sodass keine Änderungen am eigentlichen Silo vorgenommen werden müssen. Da Silos eine lange Lebensdauer von ca. 20 Jahren haben, ist die uneingeschränkte Montage des Sensors über Magnete an der Siloaußenwand eine komfortable Lösung. Dieser Aspekt spiegelt sich im Kaufpreis wider. Der Einsatz derselben Sensoreinheit für mehrere Silos ist denkbar. Da die Sensoreinheit auf der Außenseite des Silos montiert wird, ist das Gehäuse zwar den Umwelteinflüssen ausgesetzt, jedoch nicht der stark abrasiven, druckbehafteten Bestrahlung durch Feststoffpartikel. Voraussichtlich ist die Lebensdauer von Silopic länger als die von konventionellen Systemen. Die mobile funkbasierte Kommunikationsfähigkeit der Sensoreinheit ermöglicht einen hohen Automatisierungs- und Vernetzungsgrad bei der Bestellung und Bereitstellung wesentlicher Baustoffe. Dies kann die Effizienz und damit die Rentabilität von Bauprojekten erheblich steigern.

\section{Analyse der akustischen Impulsantwort}

Die akustische Impulsantwort ist eine konstant gedämpfte Schwingung und gemäß DIN 1311 [12] als transiente Schwingung zu kategorisieren. Diese entsteht bei einer sprung- oder impulsartigen Anregung und zeichnen sich durch ein chaotisches Schwingverhalten zu Anfang aus (vgl. Abb. 3). Anschließend stellen sich typischerweise geordnete Grund- und harmonische
Oberschwingungen ein. Eine spezifische Schwingung einer gewissen Frequenz kann dabei am Erregungsort punktuell abklingen, während sie entlang der Mantelfläche wandert. Kommt es nun an einer Grenzfläche wie z. B. einer Schweißnaht oder Füllstandgrenzfläche zu einer Reflektion der Schallwelle, so kann am Erregungsort ein Aufklingen dieser spezifischen Schwingung beobachtet werden. Somit ist das Frequenzspektrum der akustischen Impulsantwort über die Zeit zu beobachten.

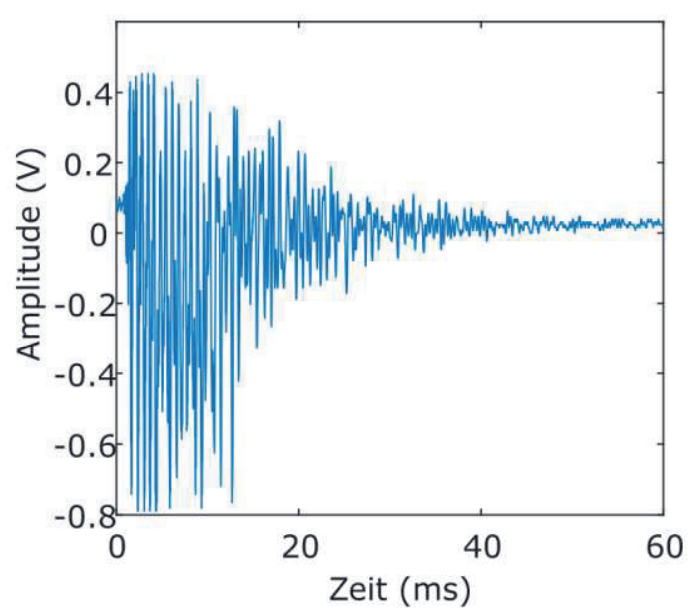

Abb. 3: Impulsantwort eines befüllten Silos

Hierzu werden drei Messverfahren in Erwägung gezogen:

- Beschleunigungssensor

- Mikrofon

- Akustische Kamera

Heutige Beschleunigungssensoren arbeiten in der Regel piezoelektrisch oder kapazitiv und können zur Messung von Körperschall eingesetzt werden (vgl. Abb. 4). Die piezoelektrischen Beschleunigungssensoren verfügen über eine seismische Masse, die an einem piezokeramischen Sensorplättchen befestigt ist [4]. Dieser Piezowiderstand wandelt dynamische Druckschwankungen in elektrische Signale um.

Kapazitive Beschleunigungssensoren sind in der Regel Mikro-Elektro-Mechanische S(MEMS). Diese Feder-Masse-Systeme werden in der Regel aus Silizium hergestellt [4]. Dabei wird eine $\mathrm{H}$-förmige Masse von vier fest verankerten Stegen in der Funktion als tragende Feder gehalten (vgl. Abb. 4). Durch die Bewegung der Masse findet eine relative Verschiebung zwischen dieser Masse und der unbeweglichen Bezugselektroden statt, welche durch die lineare Änderung der Kapazität erfasst werden kann [4]. 
a)

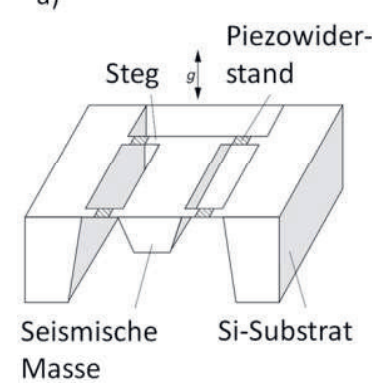

Abb. 4: a) Beschleunigungssensor, b) MEMS [13]

Gängige Mikrofone lassen sich heutzutage weitestgehend in fünf Kategorien eingliedern [14]:

- Kontaktwandler

- Piezoelektrisches Mikrofon

- Elektrodynamisches Mikrofon

- Elektrostatisches Mikrofon

- Hitzedraht-Mikrofon

Allesamt eignen sich üblicherweise zur Messung von Luftschall im Frequenzbereich zwischen $20 \mathrm{~Hz}$ bis $20 \mathrm{kHz}$. Die unterschiedlichen Messprinzipien seien in diesem Rahmen nicht näher erläutert.

Eine Akustische Kamera besteht aus mehreren gezielt angeordneten Mikrofonen (daher auch bekannt als Mikrofonarray) [14]. Eine Schallwelle breitet sich kugelförmig von ihrem Quellort aus und trifft im homogenen akustischen Fernfeld auf jedes Mikrofon mit einer unterschiedlichen zeitlichen Verzögerung auf. Nun wird durch die Interferenzrekonstruktion der Quellort bestimmt [14] und mithilfe der bildgebenden Daten einer Kamera visualisiert. Eine akustische Kamera ist geeignet um stark abstrahlende Stellen zu detektieren.

Da ein mobiles Schüttgutsilo eine Vielzahl von inhomogenen, lokalen Versteifungen besitzt, konnte messtechnisch nachgewiesen werden, dass die akustische Impulsantwort eine starke Ortsgebundenheit aufweist. Silopic muss jedoch gewisse Toleranzen hinsichtlich der Montageposition kompensieren können. Daher wurde auf die Verwendung von Beschleunigungssensoren für den Prototyp verzichtet. Bei der Verwendung der akustischen Kamera war die Positionierung außerhalb des inhomogenen akustischen Nahfeldes nicht möglich, da die Störfaktoren aus der Umgebung die akustische Impulsantwort zu stark überlagerten. Daher konnte lediglich im Nahfeld festgestellt werden, dass die beiden vertikalen Enden des senkrechten Silos eine hohe Abstrahlungsenergie aufweisen (vgl. Abb. 5).

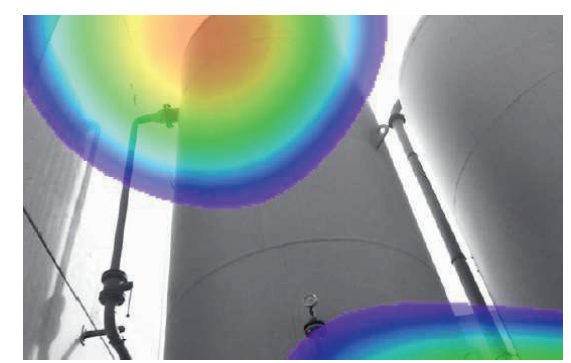

Abb. 5: Analyse des vertikalen Silos mit der akustischen Kamera

Für den Silopic-Prototyp eignet sich vor allem ein elektrostatisches Mikrofon. Diese sind preiswert, können die akustische Impulsantwort mit ausreichender Qualität messen und weisen trotz der Positionierung im Nahfeld eine gewisse Toleranz hinsichtlich der Montageposition auf.

$\mathrm{Da}$ die Messung an einem mobilen Schüttgutsilo im Realeinsatz eine gewisse Komplexität mit sich zieht, werden die Messungen im Rahmen dieser Arbeit an einem Prüfstand in Laborumgebung mit konstanter Temperatur und ohne weitere wesentliche Störeinflüsse untersucht (vgl. Abb. 6).

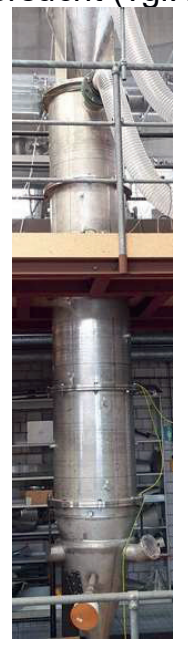

\section{Abb. 6 Silo-Prüfstand}

Die Frequenzverteilung oder das Spektrum eines Zeitsignals kann in den meisten Fällen ohne weiteres nicht beurteilt werden. Daher ist ein geeignetes Spektralanalyseverfahren erforderlich. Die bekannteste Spektralanalyse ist die Fouriertransformation (FT), die sich wiederum in vier verschiedene Verfahren untergliedert [15].

Die FT approximiert im Grunde ein kontinuierliches Zeitsignal in ein kontinuierliches Spektrum, wobei das Signal als eine Summe von periodischen und stationären Sinus oder Kosinus unterschiedlicher $\quad$ Frequenzen und unterschiedlicher Amplituden rekonstruiert wird [16]. Da die Fouriertransformierte zwar eine 
Lokalität im Frequenzbereich aber nicht im Zeitbereich hat, ist die Voraussetzung für die Approximation die stationäre Periodizität des Zeitsignals [15]. Da in diesem Anwendungsfall eine auf- und abklingende und damit eine instationäre Schwingung vorliegt, ist somit die Verwendung der FT unzulässig [12] [15] [16].

Für instationäre Signale kann die KurzzeitFouriertransformation (zu engl. Shhort-Time Fourier Tranformation [STFT]) geeignet sein [15] [16]. Dabei wird nicht das gesamte Signal fouriertransformiert, sondern mittels eines Fensters in mehrere Teilsegmente unterteilt und anschließend jedes Teilsegment quasi unabhängig analysiert.

Dadurch wird zusätzlich zur Frequenzauflösung eine grobe Zeitauflösung gewonnen [16]. Durch eine geeignete Fensterung können sogenannte Leck-Effekte [16] kompensiert werden. Die STFT eignet sich für Signale, die über die Zeit nur allmähliche Veränderungen im Spektrum aufweisen. Bei einer Impulsanregung klingt jedoch die Schwingung aus dem Ruhezustand rapide auf und unmittelbar danach wieder ab. Signale mit einer derartigen Dynamik können mit einer STFT nur sehr eingeschränkt analysiert werden, da i. d. R. weiterhin eine stationäre, periodische Funktion als Basisfunktion verwendet wird. Als Ausnahme sei hier die Gabortransformation genannt, eine Sonderform der STFT, die starke Analogien zur Wavelettransformation (WT) aufweist [15].

Die WT ist eine lineare Zeit-FrequenzTransformation, die oft als eine „Verbesserung der STFT" gesehen wird [15]. Obwohl die Grundidee bereits 1910 von Haar beschrieben wurde, nahm sie erst seit 1990, nach Entdeckung von kompakten, stetigen und orthogonalen Wavelets durch Daubechies 1988 und die Entwicklung der schnellen WT 1989, Einzug in die breite Anwendung [16]. Eine Wavelet ist eine im Grunde beliebige Funktion, die eine wellenartige Oszillation aufweist und zu Anfang und Ende gegen null läuft (vgl. Abb. 7) [16]. Die meisten Wavelets haben keine geschlossene Form. Bei der WT wird die gewählte Wavelet, die sich voraussichtlich am besten zur Approximation eines spezifischen Rohsignals eignet, als Basisfunktion verwendet. Für die hier vorliegende rasch auf- und abklingende Schwingung wird die Morlet-Waveletfunktion gewählt.

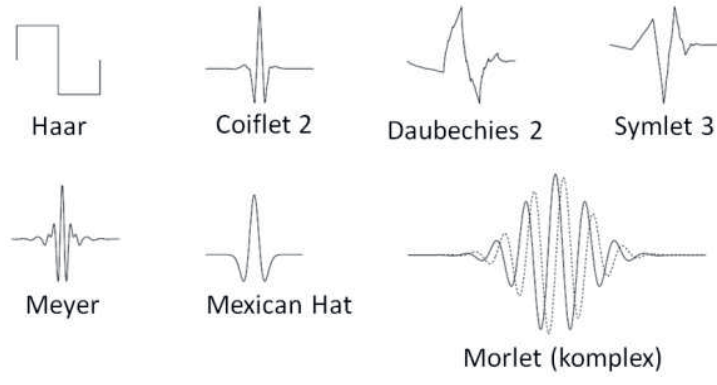

Abb. 7: Bekannte Waveletfunktionen [16]

\section{Kennwertdefinition}

Primäres Ziel der experimentellen Untersuchungen war es, den Einfluss des Schüttgutfüllstandes auf die Impulsantwort in Erfahrung zu bringen. Anschließend ist ein Kennwert zu definieren, der ausreichend mit dem Füllstand korreliert. Hierzu wurden zwei Messreihen durchgeführt, wobei jeweils das volle Silo auf dem Prüfstand gleichmäßig entleert wurde. Zwischen den beiden Messreihen wurde der Sensor um $3 \mathrm{~cm}$ verschoben, um eine Aussage hinsichtlich der Robustheit gegenüber Montagetoleranzen treffen zu können.

Abb. 8 zeigt den Vergleich zwischen der Impulsantwort eines a) vollen und b) leeren Silos. Über die Abszisse ist die Zeit in $\mathrm{ms}$ und über die Ordinate die Frequenzverteilung bei 0-16 kHz dargestellt. Die Dämpfung durch das Schüttgut (vgl. Abb. 8a)), die mit zunehmender Frequenz stärker wird, ist offensichtlich. Die Impulsantwort klingt bei einem vollen Silo nach ca. $30 \mathrm{~ms}$ ab, wobei die Schwingung bei einem leeren Silo (vgl. Abb. 8a)) zum gleichen Zeitpunkt lediglich ein wenig gedämpft ist.

Um aus den transformierten Daten einen zulässigen Füllstands-Kennwert zu berechnen, werden für unterschiedliche Zeit- und Frequenzbereiche die sieben gängigsten statistischen Kenngrößen ermittelt (Mittelwert, Median, Minimum, Maximum, Modus, Streuung, Varianz) [17].

Dabei zeigt der Medianwert im Zeitbereich 11$14 \mathrm{~ms}$ nach dem Schlag und in einem Frequenzbereich von $2500-5000 \mathrm{~Hz}$ eine deutliche Sensitivität hinsichtlich des Füllstandes zwischen $100 \%$ und $95 \%$ und zwischen $40 \%$ und $0 \%$ (vgl. Abb. 9). Bei einem Füllstand zwischen $95 \%$ und $40 \%$ ist die Aussagekraft des Kennwertes aufgrund der großen Streuung gering. 


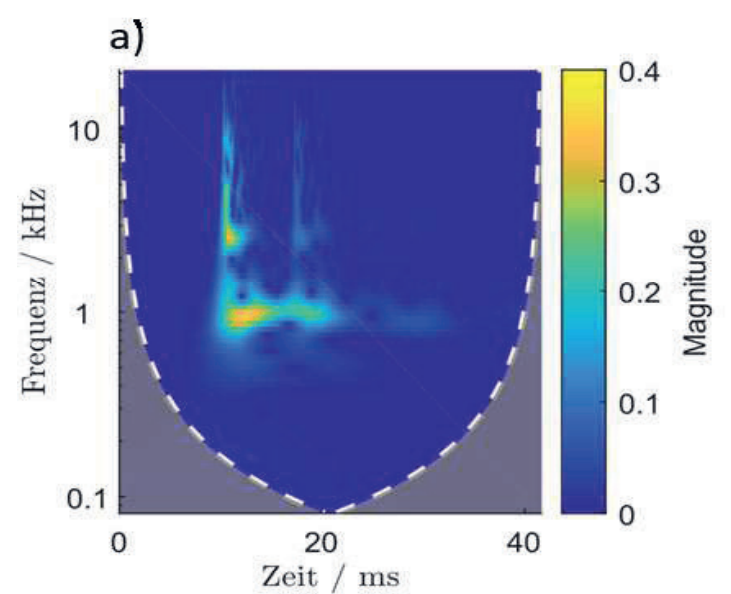

b)

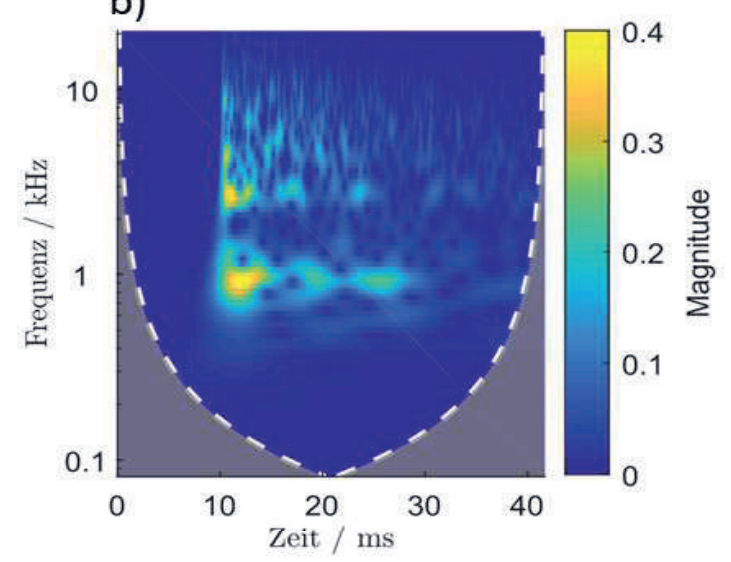

Abb. 8: Akustische Impulsantwort eines a) vollen Silos und b) eines leeren Silos

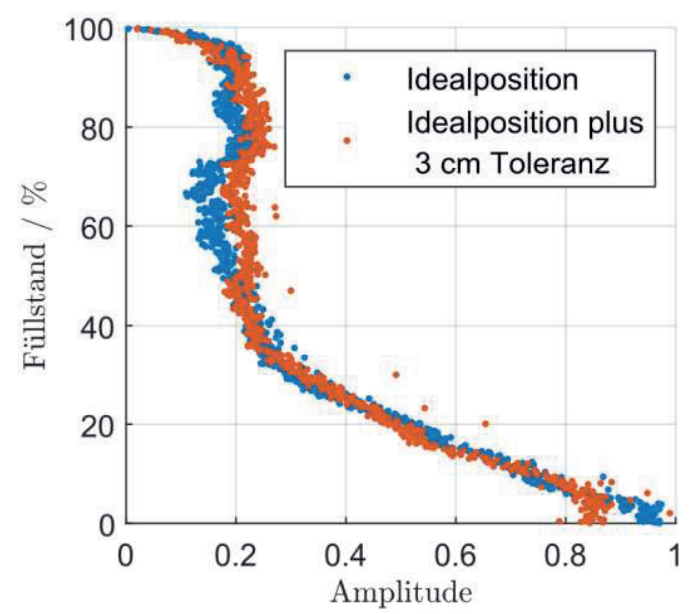

Abb. 9: Verlauf des Silopic-Kennwertes im Frequenzbereich von $2500-5000 \mathrm{~Hz}$ und Zeitbereich von 11 - 14 ms nach Impulsanregung

\section{Zusammenfassung}

Im Rahmen dieser Arbeit wurde der heutige Stand der Füllstandmesstechnik für Schüttgüter erläutert. Weiterhin wurde ein neues Konzept für einen akustischen
Füllstandsensor zur Anwendung an mobilen Schüttgutsilo präsentiert. Die Eignung verschiedener Sensoren wie Beschleunigungssensoren, Mikrofone oder akustische Kameras wurden beurteilt. Zur Analyse der Impulsantwort wurde eine geeignete Methode der Signalverarbeitung gewählt. Erste experimentelle Untersuchungen haben den Einfluss des Füllstandes auf die akustische Impulsantwort eines Silos auf dem Prüfstand gezeigt. Auf Basis dieser Daten konnte erstmalig ein prototypischer Kennwert berechnet werden, der hinreichend mit dem Schüttgutfüllstand korreliert.

\section{Literaturnachweis}

[1] Der Joker im Silo; PROCESS; url: https://www.process.vogel.de/der-joker-im-siloa-55399/; zugegriffen am 08.05.2019

[2] K. W. Bonfig, A. Liske; Fullstand-Meßtechnik; Hans Holzmann Verlag KG, (1973)

[3] E. Hering, H. Steinhart; Taschenbuch der Mechatronik; Carl Hanser Verlag, (2015); ISBN: 978-3-446-43857-6

[4] G. W. Schanz; Sensoren - Fühler der Meßtechnik; Dr. Alfred Hüthig Verlag Heidelberg, (1988)

[5] G. Strohrmann; Meßtechnik im Chemiebetrieb; R. Oldenburg Verlag München Wien, (1995); ISBN: 3-486-22999-0

[6] M. Rohn; Füllstandmessung mit Ultraschall; Endress+Hauser, (2014)

[7] H. Bohlmann, S. Mühlenkamp; Wie das Radar Schüttgut lernte; PROCESS; url: https://www.process.vogel.de/wie-das-radarschuettgut-lernte-a-421144/; zugegriffen am 08.05.2019

[8] Der Radarsensor für Schüttgüter VEGAPULS 69; VEGA, (2014)

[9] M. J. Heim, A. Mayr, Endress+Hauser GmbH, (2001)

[10] Geführtes Radar: VEGAFLEX Serie 80; VEGA, (2015)

[11] L. Anslinger, P. Sivasothy, F. Torner, J. Seewig; Mobile sensor concept for acoustic level estimation in bulk solids silos; IEEE Sensors Applications Symposium (SAS), (2018); doi: 10.1109/SAS.2018.8336760

[12] Schwingungen und schwingungsfähige Systeme; Deutsches Institut für Normung; (2000); DIN 1311-1

[13] W. Schmidt; Sensorschaltungstechnik; Vogel Buchverlag, (2002); ISBN: 978-3-8343-3111-3

[14] M. Eichler; Breitbandige BeamformingAlgorithmen zur Erfassung von Audiosignalen mit kompakten Mikrofon-Arrays; Dissertation, (2009)

[15] A. Mertins; Signaltheorie, Springer Verlag, (2013); doi: 10.1007/978-3-8348-8109-0

[16] S. Tamm; Hochaufgelöste Zeit-FrequenzAnalysen ereigniskorrelierter EEGOszillationen mittels S-Transformation; Dissertation, (2005)

[17] L. Fahrmeir, R. Künstler, I. Pigeot, G. Tutz; Statistik; Springer Verlag, (2007); ISBN: 978-4540-69713-8 